eISSN: 2659-6482

DOI: https://doi.org/10.14201/pmrt.24485

\title{
LA ACTIVIDAD MUSICAL EN FUENTE LIBRILLA. RECORRIDO HISTÓRICO A TRAVÉS DE LOS PROGRAMAS DE FIESTAS
}

\section{The Musical Activity in Fuente Librilla. Historical Journey through the Party Programs}

\author{
Norberto LÓPEZ NÚÑEZ \\ (Universidad de Murcia) \\ norberto.lopez@um.es
}

RESUMEN: El presente estudio tiene por finalidad identificar y analizar desde el punto de vista histórico la actividad musical presente en Fuente Librilla en las últimas tres décadas (1989-2019) a través de los programas de fiestas. Los programas de fiestas son publicaciones editadas de forma amateur a través de los servicios de edición de una imprenta y que carecen de depósito legal e ISBN. Desde el punto de vista metodológico, se ha realizado un estudio cualitativo de corte documental. Los resultados revelan la presencia de más de 154 agrupaciones de diversa índole relacionadas con la música. Principalmente de grupos u orquestas musicales para amenizar la verbena popular y propiciar el baile.

Palabras clave: Fuente Librilla; actividad musical; programas de fiestas; fiestas patronales.

ABSTRACT: The present study aims to identify and analyze from the historical point of view the musical activity present in Fuente Librilla in the last three decades (1989-2019) through party programs. The party programs are publications published in an amateur way through the publishing services of a printing company and without legal deposit and ISBN. From the methodological point of view, a qualitative documentary study has been carried out. The results reveal the presence of more than 154 groups 
of various kinds related to music. Mainly of groups or musical orchestras to liven up the popular verbena and promote dancing.

Keywords: Fuente Librilla; Musical Activity; Party Programs; Patron Saint Festivities.

\section{INTRODUCCIÓN}

Fuente Librilla es un pequeño pueblo situado a las faldas de Sierra Espuña y perteneciente al término municipal de Mula, ubicado en la comarca del Río Mula de la Comunidad Autónoma de la Región de Murcia (España). Es una pedanía rica, desde el punto de vista de acontecimientos relacionados con el hecho sonoro-musical, por la gran variedad de tradiciones y actividades musicales que se desarrollan. Desde cánticos de alabanzas de origen popular religioso atesorados por su antigua Campana de Auroros, pasando por los toques de campana y carraca, hasta la tradicional carrera de aguilandos o bailes de puja que tienen lugar en fechas navideñas. Podríamos decir que es un lugar destacado donde el folklore musical aún sigue vivo. No obstante, nuestro interés de estudio no se centra en analizar los acontecimientos musicales relacionados únicamente con el folklore musical de forma aislada, sino el estudio de la actividad musical desarrollada a lo largo del tiempo durante las fiestas patronales a través de los programas de fiestas realizados para la ocasión.

Las fiestas patronales de Fuente Librilla tienen lugar durante el primer o segundo fin de semana del mes de octubre cuya proximidad esté más cercana al día 7 de octubre, coincidiendo con el día de la Virgen del Rosario, patrona local en cuyo honor se celebran las fiestas. Desde finales de la década de los años ochenta del siglo XX hasta la actualidad, la duración de las fiestas ha sido de 5 días, comenzando en viernes y finalizando en el martes siguiente. Esto no ha sido siempre así, se tiene constancia a través de programas de fiestas de la década de los años 40 y 50 del siglo XX de que las fiestas se organizaban durante solo tres días, siendo estos sábado, domingo y lunes. La gestión y organización de las fiestas patronales se ha venido desarrollando por la voluntad y altruismo de personas afines (grupos de amigos, peñas, familias, quintos) que, de manera desinteresada y en pro del interés colectivo para sufragar en gran medida los costos de las mismas, trabajan durante todo un año previo a la celebración de las fiestas organizados en un grupo denominado «Comisión de Fiestas o de Festejos». Dentro de estos cinco días de fiesta, tienen cabida multitud de actividades y eventos donde la música adquiere un papel protagonista: pasacalles de bandas o charangas, toques de campanas, conciertos de música, verbenas musicales, bailes, encuentros de cuadrillas o artistas de diversa índole. 
El objetivo principal del presente estudio es identificar y analizar desde el punto de vista histórico la actividad musical presente en Fuente Librilla en las últimas tres décadas (1989-2019) a través de los programas de fiestas.

Desde el punto de vista metodológico, se ha realizado un estudio cualitativo de corte documental donde se han analizado todos los programas de fiestas de Fuente Librilla desde el año 1989 hasta 2019. Además, se han tenido en cuenta otras fuentes documentales como monografías, prensa histórica y publicaciones de carácter local.

Las fuentes para este estudio las constituyen un total de 39 documentos correspondientes a los programas de las fiestas patronales de Fuente Librilla comprendidos entre los años 1989 y 2019. Además, se ha podido tener acceso a los programas de fiesta de los años 1924, 1944, 1946, 1950, 1957, 1982, 1984, 1986 y 1988.

Los programas de fiestas son publicaciones editadas de forma amateur a través de los servicios de edición de una imprenta y que carecen de depósito legal e ISBN. $\mathrm{Su}$ estructura interna alberga varias secciones bien diferenciadas. La primera de ellas está conformada por unas páginas preliminares donde se encuentran el índice, saludos de forma individualizada por las autoridades religiosas, civiles, locales y la propia comisión de festejos. La segunda sección está destinada a la descripción detallada de la programación de fiestas distribuida a lo largo de los cincos días que dura la fiesta, donde se detallan fechas, horarios, tipo de actividad y lugar de celebración, así como información complementaria sobre la realización de las actividades. También, en ocasiones, suele haber una programación con la descripción de los mismos elementos (fechas, horario, lugar y tipo de actividad) de unas actividades previas a los días centrales de la fiesta denominadas «prefiestas». La segunda sección suele colocarse en las páginas centrales del programa de fiestas y sobre esta se centra nuestro análisis de estudio. Una tercera sección, la más extensa, está destinada a los anuncios publicitarios de empresas, comercios, servicios o entidades de carácter local o de localidades vecinas a Fuente Librilla. Esta sección se distribuye en dos partes: entre las páginas preliminares y la programación de fiestas; y después de la programación de fiestas hasta el final. En ocasiones suele haber una cuarta sección destinada a pequeños reportajes fotográficos, artículos de opinión o divulgación, poesías o relatos relacionados con el propio pueblo. Su ubicación y extensión ha variado a lo largo de los años, pero por lo general suele estar ubicada hacia final del programa de fiestas. Debemos destacar también que desde principios de la década de los años noventa del pasado siglo y hasta la actualidad la extensión de los programas se ha mantenido en torno las 100 y 200 páginas ${ }^{1}$. Por el contrario, desde principios del siglo XX hasta final de los años

1. Esto es debido a la gran cantidad de anunciantes que figuran en el mismo y con cuyas aportaciones económicas a cambio de publicitarse se sufragan los gastos de la edición de los ejemplares. Su distribución es gratuita entre todos los habitantes del pueblo. 
ochenta, los programas de fiestas eran de menor extensión, aunque mantenían las tres secciones principales (páginas preliminares, programación y anuncios).

\section{ANTECEDENTES}

Con la intención de trazar una línea sobre los estudios previos al tema que nos ocupa, sirvan los párrafos siguientes como estado de cuestión, dado que no se ha encontrado ningún estudio similar que aborde en profundidad el tema en cuestión.

Sobre aspectos musicales de Fuente Librilla podemos encontrar varios libros y artículos relacionados con el folklore musical cuya temática versa sobre los auroros de Fuente Librilla (López, 2012) o aspectos relacionados con el hecho musical del lugar de forma general (López, 2015b; López, 2016), así como un cancionero popular de la localidad (López, 2015a). Otro aspecto del folklore musical tratado sobre Fuente Librilla han sido las aportaciones de García (2012a) en torno a los bailes de pujas o las carreras de aguilandos celebrados en fechas navideñas.

Con relación al estudio de la actividad musical en las fiestas patronales son escasos los estudios hallados. Aramburu (1987) describe el baile en las fiestas patronales de dos pueblos navarros desde una perspectiva social. Botella (2015) realiza un análisis documental de la actividad musical realizada en las Fiestas de Moros y Cristianos de la localidad de Alcoy (Alicante) a través de los programas de fiestas de la revista anual de la fiesta local. Valverde (2017), como cronista oficial de Campos del Río, destaca en su artículo que su pueblo no tiene banda de música, pero sí han pasado por él muchas bandas, de las cuales se va a dar cuenta de ello a través de las crónicas recogidas en la prensa escrita entre los años 1916 a 1961. También destaca que solo se hace mención a las bandas que han actuado en el marco de las fiestas patronales de Campos del Río.

\section{ACTIVIDAD MUSICAL}

\subsection{Bandas}

La primera noticia que tenemos constancia de la participación de una banda de música en las fiestas patronales de Fuente Librilla se remonta al año 1900. El diario murciano Las Provincias de Levante del 10 de octubre del citado año menciona la participación de la banda de Alcantarilla ${ }^{2}$ y elogia a su maestro D. Francisco Gómez $z^{3}$ por la interpretación de una malagueña con su requinto

2. P. (10 de octubre de 1900). En Fuente Librilla. Las Provincias de Levante, p. 1.

3. Sánchez (2017: 79) afirma que fue director de la banda de Alcantarilla vinculada al Casino en 1898 y que en 1900 ocupó la dirección de la citada banda de música don José Hita. 
durante las fiestas patronales ${ }^{4}$. También se tiene constancia de la participación de la banda de Pliego en 1906 en las fiestas de Fuente Librilla (García, 2012b). Las bandas de música que han actuado en Fuente Librilla durante sus fiestas provienen de localidades vecinas tales como Librilla, Pliego, Mula, Alcantarilla o Aledo, todas ellas de localidades colindantes al pueblo a excepción de Aledo y Alcantarilla. Su principal cometido ha sido y es el acompañamiento durante el desfile procesional que tiene lugar el domingo central de las fiestas en torno a las 20:00 horas. Desfile procesional que se realiza en honor a la Virgen del Rosario, patrona local. Esta actividad ha sido llevada a cabo en la mayoría de ocasiones por las bandas de música de Mula ${ }^{5}$ y Pliego. Entre otras actividades con menor participación cabe destacar pasacalles de alborada, desfiles de carrozas, romerías hacia el barrio de Los Cortijos o actuaciones para amenizar el baile en la verbena popular. Son muy pocos los conciertos realizados por las bandas en el marco de las fiestas patronales, algunos de ellos, los realizados entre los años 1944 a 1957, pudieran considerarse actuaciones para amenizar el baile durante la verbena popular. No obstante, en los programas de fiestas correspondientes a los años 1944, 1946, 1950 se destaca la presencia de Baltasar Espada Cánovas ${ }^{6}$ como director de la banda de música de Librilla, donde además de pasacalles realiza conciertos para amenizar una verbena. En este sentido, Barquero (2017: 392) afirma que «la banda de Librilla hacía los festejos en muchos pueblos de la región por su gran calidad musical, era importante y conocida. Las fiestas de Sangonera la Verde, de Fuente Librilla, Barqueros, El Berro...».

4. Dado que las crónicas del diario Las Provincias de Levante a fecha del 10 de octubre de 1900 ponen de manifiesto que Francisco Gómez Tomás estaba al frente de la banda de Alcantarilla hasta ese mismo mes, es posible que lo hiciese hasta el cambio de nombramiento de José Hita en ese mismo año. Por tanto, Francisco Gómez Tomás estuvo al frente durante poco menos de dos años.

5. Debemos destacar la forma de nombrar a la banda de Mula, desde 1984 en adelante se nombra como banda del «Excelentísimo Ayuntamiento de Mula o Municipal de Mula». Es curioso el trato de municipalidad que se le da a la banda cuando según López (2017: 448) la banda municipal de Mula se disolvió oficialmente en 1973. Probablemente su tratamiento se deba al desconocimiento por parte de la sociedad de Fuente Librilla del papel que desempeña la banda de música de Mula desde 1974 hasta la actualidad.

6. Según Barquero (2017: 390) aparece como director aprobado por oposición de la banda municipal de Librilla en el Boletín de la Asociación Nacional de Directores de Bandas de Música Civiles con fecha del 15 de mayo de 1936. 
NORBERTO LÓPEZ NÚÑEZ

LA ACTIVIDAD MUSICAL EN FUENTE LIBRILLA. RECORRIDO HISTÓRICO A TRAVÉS

DE LOS PROGRAMAS DE FIESTAS

\subsection{Charangas}

Desde $1986^{7}$ hasta la actualidad se tiene noticia de la participación de cinco charangas musicales que han puesto música en las fiestas de Fuente Librilla: Los Geranios (1986-1991), Los Colores (1992), Txaranga Amazonas (1994-2013), La Hucha (2014-2016, 2018-2019) y La Timba (2017). Entre sus principales funciones está la de tocar en los distintos pasacalles vespertinos realizados en casi todos los días de la celebración de las fiestas para despertar al pueblo recorriendo las calles del mismo entre las 9:00 y las 10:00 horas de la mañana, sirviendo estos en ocasiones para la recogida de reinas de fiestas y autoridades (miembros de la comisión de fiestas o alcalde pedáneo). Aunque también han realizado pasacalles por la tarde del viernes coincidiendo con el inicio de las fiestas. Otra actividad a destacar es la participación en la tradicional «carrera de cintas» ${ }^{8}$ poniendo música (pasodobles) cuando un joven consigue coger una de las cintas. La realización de romerías al barrio de Los Cortijos, amenizar el ambiente para otro tipo de actividades como comidas populares o poner música para bailar bailes típicos (pasodobles, valses o copla) a la salida de misa también han formado parte de su actividad musical. Por último, destacar que la actividad más relevante ha sido la participación en el desfile de carrozas que tiene lugar el martes de las fiestas por la tarde. En dicho desfile la charanga adquiere un papel protagonista como único grupo musical que toca música en directo, atendiendo en ocasiones a las peticiones musicales particulares de la gente que lo solicita. Otro de los momentos importantes durante el desfile es la canción de «El Moro», donde la charanga adquiere un mayor protagonismo cuando interpreta la canción durante al menos dos ocasiones en el recorrido del desfile y la cual la gente que participa en el mismo la baila de forma colectiva (López, 2015: 54).

Debemos destacar que la charanga Amazonas es el grupo musical que más tiempo ha actuado en Fuente Librilla, veinte años desde 1994 hasta 2013 de forma

7. Aunque en 1957 se tiene noticia de la participación en las fiestas patronales de la banda de música de la Ribera de Molina El Orfeo, dado que no se han encontrado datos de la creación de la una banda en la Ribera de Molina, es muy probable que se trate de una charanga o grupo musical festivo.

8. Actividad festiva que consiste en alcanzar unas cintas enrolladas con el nombre de una joven del pueblo que ha bordado una banda que lleva elegantemente puesta el día de la carrera de cintas. Los participantes, subidos en sus motos o motocicletas, tienen que demostrar sus habilidades y puntería para conseguir enhebrar con un palo de madera una anilla de la que cuelga a varios metros de altura la cinta enrollada en un alambre y todo ello sin poner un pie en el suelo y sin parar la moto. El que consigue cogerla es obsequiado con un beso de la joven y la colocación de su banda, además de escuchar un pasodoble tocado por la charanga en honor a su proeza. 
ininterrumpida. Es por ello que, en algunos de los programas de fiestas 9 , se alude de forma coloquial a alguno de sus integrantes o a la forma de actuar de alguno de ellos.

\subsection{Coros}

Solo tenemos constancia de la participación del coro parroquial de Fuente Librilla en las fiestas patronales en $1990^{10}$, en una misa de campaña en la barriada de Los Cortijos el viernes de las fiestas. El resto de intervenciones corales durante el periodo estudiado y en el marco de las fiestas patronales de Fuente Librilla ha sido por otros coros distinto al local. Estos han sido: Coro Villa de Alhama (2006), Coro Gosspel Eirené (2007), Coro extraparroquial San Lázaro (2017 y 2018) y Coro parroquial de Javalí Nuevo (2019). Todos los coros han participado dentro de la misa realizada en honor a la Virgen del Rosario el domingo a las 12:00 horas.

\subsection{Grupos pop-rock}

En 1989 se organiza por primera vez en Fuente Librilla un concierto de género rock donde actúan bandas de prestigio nacional como Malvariche ${ }^{11}$ o Jhonny Juerga y los que remontan el Pisuerga ${ }^{12}$. Hubo que esperar hasta el año 2003 para volver a escuchar grupos de rock en dicho lugar, esta vez grupos y artistas de renombre nacional como Fenómenos Extraños, Santi Campillo o Miguel Bañón. Es en el año 2001 cuando se organiza el I Fuente Rock en el que participan algunos de los grupos anteriores como Malvariche y Fenómenos Extraños. Diecisiete años después, en 2018, se vuelve a organizar el II Fuente Rock con la participación de grupos regionales. Cabe destacar la participación en las fiestas del grupo local Resakosis $^{13}$ en los años 2013, 2014 y 2015 en un festival denominado «Resakosis y amigos», cuya temática está destinada a la música rock, punk, entre otros géneros, en el que participan bandas y grupos de localidades vecinas junto al grupo punk anfitrión.

9. Programas de fiestas de 2003, 2005, 2006, 2008, 2010 y 2011. Casi siempre de alude al Moñoño, Antonio García García, conocido músico y miembro de la charanga Amazonas.

10. Se tiene constancia de otra intervención en el año 1957, donde se intercalaban motetes eucarísticos entre el coro parroquial y la banda de música presente durante la celebración de la misa.

11. También actúa de forma aislada en $\mathbf{1 9 9 5 .}$

12. También actúa de forma aislada en 1991.

13. Segundo grupo de música rock-punk creado en el año 2007 (López, 2015b). El primer grupo de rock de Fuente Librilla se llamaba Arte Uno y actuó en las fiestas patronales de Fuente Librilla en 1992. 
Respecto al género pop, en el año 2006 se organiza el I Fuente Pop, en el que participan los grupos y artistas vencedores de los diversos concursos organizados en la Región de Murcia, entre ellos, Audio (grupo de pop alternativo), Straperlo (grupo de pop comercial) y Juanjo Ortega (cantautor). El festival está subvencionado por el Instituto de la Juventud de la Región de Murcia. En el 2007 se organiza el II Fuente Pop de la mano de los grupos finalistas del concurso regional Murcia Joven. También debemos mencionar la participación en el año 2013 del grupo pop muleño Número 28 y del grupo pop de los 80, también muleño, Rebobina, en los años 2015 y 2018.

Cabe destacar que ambos géneros, rock y pop, únicamente han tenido lugar a través de la organización de sendos festivales (con dos ediciones cada uno) en el marco de las fiestas patronales. El día destinado para ello según los programas de fiestas analizados ha sido viernes de la fiesta principal o un día del fin de semana previo a la celebración de las fiestas cuyas actividades se enmarcan dentro de las denominadas «prefiestas». Es importante señalar que este tipo de música siempre se organiza a altas horas de la noche o incluso en la madrugada.

\subsection{Grupos musicales}

Hacia la mitad del siglo XX, la música de la verbena popular en las fiestas patronales de Fuente Librilla corría a cargo de la banda de Librilla, como así se pone de manifiesto en los programas de fiestas (1924-1957) con actuaciones tituladas «Verbena a la veneciana o música para el baile». A partir de la década de los años 80 se empieza a diversificar la participación de otros grupos para las actuaciones de la verbena. Desde 1989 hasta 2019 han actuado en las fiestas patronales de Fuente Librilla un total de 87 grupos u orquestas ${ }^{14}$ musicales distintas, destinadas a amenizar la verbena popular, con grupos de reconocido prestigio nacional como La Guardia, Los Puntos o Los Diablos. Entre los 87 grupos participantes: 16 de ellos lo han hecho en dos ocasiones, 6 en tres ocasiones, 1 en cuatro ocasiones y 3 en siete veces. Estos últimos han sido el Grupo Guadaira (1989 a 1993 y 1995), Grupo Rusadir (1989 a 1991, 1993 y 1994) y la Orquesta Zona Azul (1992, 1995, 1996, 2001 a 2003, 2005 y 2008). Incluso algunos de los mencionados grupos han actuado en más de una ocasión en el mismo año, como por ejemplo el Grupo Guadaira, que actuó dos veces en el año 1992, o el Grupo Rusadir, tres veces en

14. Los grupos musicales que actúan en verbenas suelen denominarse «orquestas» de forma errónea. Su formación instrumental no se corresponde con la definición de orquesta dentro del ámbito de la música. No obstante, dado que la terminología que emplean las fuentes consultadas alude de esta forma a los grupos musicales que actúan en ella, nosotros hemos querido respetar la terminología para no confundir. Si bien es cierto que se alude de manera indistinta a los grupos musicales que figuran en los programas de fiestas como «orquestas» o «grupos». 


\section{NORBERTO LÓPEZ NÚÑEZ \\ LA ACTIVIDAD MUSICAL EN FUENTE LIBRILLA. RECORRIDO HISTÓRICO A TRAVÉS \\ DE LOS PROGRAMAS DE FIESTAS}

1991. También han actuado otras formaciones más simples como dúos musicales en un total de 6 veces durante los 30 años, cumpliendo únicamente la función de teloneros de otros grupos. Desde el punto de vista organizativo y teniendo en cuenta que las fiestas duran cinco días desde viernes a martes, el martes es el día en el que menos grupos u orquestas han participado (18), por el contrario, hay una similitud en el resto de días con un rango de intervenciones musicales entre 30 y 32: viernes (30), sábado (32), domingo (31) y lunes (31).

\subsection{Artistas}

Entre 1989 y 1993 no ha participado ningún artista solista en las fiestas patronales de Fuente Librilla. Es a partir de 1994 cuando empiezan a actuar, pero sobre todo se hacen más partícipes en la década de 2010 en adelante. La participación de artistas solistas tiene la intención de servir de entretenimiento a distintos sectores poblacionales. Por un lado, amenizar al público de mayor edad con actuaciones de cantantes de copla española como Maribel Castillo ${ }^{15}$ o la vedete Merche Lois. Por otro lado, han actuado artistas solistas de la talla de David Andreu, Antonio Micol o Moy Gommar para un público más adulto. También se ha tenido en cuenta al público infantil con la intervención de artistas como Pepica y los Bichejos. Por el contrario, la intervención de artistas solistas también ha tenido la intención de ser un reclamo turístico. La participación de artistas o personajes relacionados con el mundo de la canción y con renombre en el panorama nacional también ha tenido cabida en las fiestas patronales de Fuente Librilla, prueba de ello son las descripciones que figuran en los programas acompañando el anuncio de la actividad en cuestión, entre ellos han actuado: Marcos Llunas (hijo del cantante Dyango), El Pulpo (locutor de Cadena 100), Angy (actriz y cantante finalista de Factor X), Sandra Bruman (vedete y tertuliana de Telecinco) y El hombre de las mil voces (Participante en Got Talent).

\subsection{Disc jockeys}

La actividad musical relacionada con los disc jockeys (DJ) en las fiestas de Fuente Librilla ha tenido lugar en la última década ininterrumpidamente (20102019). En todas las programaciones de fiestas de los años anteriormente citados podemos encontrar la presencia de cinco DJ: DJ Pana, DJ Juan Ruiz ${ }^{16}$, DJ Vinilo, DJ Jaro y DJ Chumi. Su actividad se ha centrado en poner música a altas horas de la madrugada como actividad de continuación tras el final de la actuación del

15. Ganadora del exitoso programa de 7 Región de Murcia A tu Vera.

16. DJ Pana y DJ Juan Ruiz son dos disc jockeys de Fuente Librilla que han actuado en varias ocasiones (2010, 2012 y 2013) en un cara a cara musical. 


\section{NORBERTO LÓPEZ NÚÑEZ \\ LA ACTIVIDAD MUSICAL EN FUENTE LIBRILLA. RECORRIDO HISTÓRICO A TRAVÉS \\ DE LOS PROGRAMAS DE FIESTAS}

grupo u orquesta de un determinado día. También se han encargado tradicionalmente de poner música el último día de las fiestas, martes.

\subsection{Grupos folklóricos}

Son numerosos los grupos folklóricos que se señalan en los programas de fiestas de Fuente Librilla. Todos ellos, a excepción del grupo local ${ }^{17}$, son pertenecientes a peñas huertanas de Murcia o Mula y su actividad se ha mantenido relativamente constante a los largo de los años analizados. Asimismo, cabe destacar la participación del grupo de coro, danzas y rondalla de la Peña La Zaranda del Barrio de San Antón de Murcia durante seis ocasiones ${ }^{18}$. De la huerta de Murcia también han actuado los grupos folklóricos de las peñas Virgen de la Arrixaca (1989), El Salero (2001), La Esparteña (2003) y El Almendro (2009). Los grupos folklóricos de las peñas huertanas de Mula han actuado en mayor número de ocasiones debido a la vinculación al pueblo de Fuente Librilla por ser pedanía de la misma. Cabe destacar la participación del grupo de coros y danzas del Niño Jesús de Balate de la peña huertana Alegría Muleña en seis ocasiones ${ }^{19}$. También ha actuado el grupo folklórico de la peña huertana de Mula Los Muleñicos en un total de dos ocasiones (1998 y 2000). Entre las principales funciones de los grupos folklóricos dentro de la programación de fiestas estaba la de acompañar la ofrenda floral del sábado, participar en la misa del domingo y la de realizar demostraciones de bailes típicos regionales.

\subsection{Cuadrillas de ánimas}

Fuente Librilla cuenta con una cuadrilla de ánimas que rinde honores a la Virgen del Rosario y organiza desde el año 2007 un encuentro de cuadrillas regional con gran interés y participación por parte de otras cuadrillas de ánimas y grupos folklóricos de la región de Murcia y fuera de ella. Fruto de ello, en algunos programas de fiestas $(2007,2010$ y 2011) se dedican algunas páginas a reportajes fotográficos sobre el encuentro y algún artículo de divulgación del mismo. Su participación dentro de las fiestas patronales se centra en poner música y cante en dos actos religiosos: la ofrenda floral a la Virgen del Rosario y la misa del día posterior en la que acompañan cantando las distintas partes de la misa. En ocasiones (2009-2013), han participado conjuntamente en los actos religiosos

17. Denominada Rondalla de Pulso y Púa de Fuente Librilla y dirigida por el maestro Francisco Hernández (Comisión de Fiestas, 1993), ha actuado en cuatro ocasiones correspondientes a los años 1992, 1993, 1994 y 1995.

18. En los años 1990, 1991, 1995, 2005, 2013 y 2014.

19. En los años 1992, 2006, 2007, 2008, 2017 y 2018. 
cantando con la Cuadrilla de las Benditas Ánimas de Patiño, cuadrilla hermanada con la cuadrilla de Fuente Librilla. Debemos matizar que, además de aparecer las referencias a la cuadrilla en la programación general de las fiestas, también se menciona en la programación de actos religiosos dentro de algunos programas de fiestas (2010 y 2011).

\subsection{Troveros}

El mundo del trovo ha estado ligado a Fuente Librilla desde la recuperación de tradiciones como el baile de puja o la carrera de aguilandos. De vital importancia ha sido la figura de Manuel Cárceles, el Patiñero. No obstante, la presencia de veladas de trovo y la participación de troveros en las fiestas de Fuente Librilla también han quedado recogidas en los programas de fiestas. Ilustres troveros murcianos se han dado cita en el marco de las fiestas a través de veladas de trovo, misas cantadas, versos dentro del desfile procesional o incluso con pregones de fiestas. Entre ellos destacamos al Patiñero, el Reputín, el Taxista, el Conejo II, el Cardoso, el Cardoso II, el Andaluz, Salva (trovero de Fuente Librilla) y el Floristero (pregonero de las fiestas 2009).

\subsection{Campanas}

El uso de las campanas del campanario de la iglesia parroquial ha tenido un papel relevante dentro de las fiestas locales en Fuente Librilla. Se tiene constancia de ello gracias a las indicaciones de todos los programas analizados, donde hasta 1950 no se hacía referencia a la hora exacta del toque, simplemente se indicaba que era al amanecer. Es a partir de esa fecha cuando se empiezan a indicar las horas de toque. Desde 1989 hasta $2010^{20}$ se han venido tocando las campanas prácticamente todos los días de las fiestas al amanecer en determinadas horas entre las 8:00 y las 9:30 de la mañana. Entre los motivos indicados podemos encontrar: despertar al pueblo, toques de alborada o toques por la tarde, concretamente el viernes, para indicar el inicio de las fiestas. Debemos destacar que desde el año 2000 hasta el 2010 la hora de toque se ha ido retrasando entre las 10:00 y las 11:00 horas. Es importante señalar que al inicio de la década de los años 10 del presente siglo, su uso no se ha reflejado en los programas de fiestas, únicamente hay tres alusiones a los años 2011, 2018 y 2019 en un único día (viernes) coincidiendo con el inicio de las fiestas a las 17:00 horas. Respecto a su uso, se indica generalmente el volteo de campanas y en

20. A excepción del año 2006, que creemos que sí se tocaron por la tendencia estudiada, pero no se plasmó en el programa. 


\section{NORBERTO LÓPEZ NÚÑEZ \\ LA ACTIVIDAD MUSICAL EN FUENTE LIBRILLA. RECORRIDO HISTÓRICO A TRAVÉS \\ DE LOS PROGRAMAS DE FIESTAS}

menor medida el replique siempre de forma manual. Esto puede deberse a que el mecanismo de martillo y volteo está en desuso (García, 2003).

\subsection{Baile y danza}

El baile dentro de los programas de fiestas se menciona para referirse principalmente a dos actos diferenciados. En la década de 1990 hasta el 2000 el baile se materializa a través de las actuaciones de los y las jóvenes del pueblo mediante el baile de sevillanas en honor a la Virgen tras la ofrenda floral o como actuación en el barrio de Los Cortijos ${ }^{21}$. Aunque a partir del año 2000 no se vuelve a realizar este tipo de actividades de forma sistemática, sí se realizan de forma aislada durante los años 2006 y 2008. Además se advierte de la presencia de los profesores $^{22}$ de baile (sevillanas) durante la actuación. El segundo de los actos reflejado en los programas de fiestas es para referirse a los bailes que tienen lugar a la salida de la misa del domingo en la plaza de la iglesia. En dichos actos, la charanga de turno suele ser la encargada de interpretar piezas bailables para los asistentes (pasodobles), aunque en ocasiones también lo ha hecho la cuadrilla local (2010).

Desde el punto de vista de la danza, entendida como baile organizado con coreografía establecida, las únicas evidencias en los programas de fiestas son las realizadas a las comparsas durante el desfile de carrozas que tiene lugar el martes. Dos son las comparsas que han actuado en Fuente Librilla, Las Divinas (2011) y la comparsa Nuevo Encanto en tres ocasiones (2014, 2016 y 2018) ${ }^{23}$. Destacar que también hay un gran número de referencias a la participación de gogós durante el desfile del martes acompañadas por una discoteca móvil. Por último, se mencionan de forma aislada tres actuaciones relacionadas con la danza: el Ballet SIKO (finalista en el concurso televisivo de Bravo Bravísimo) en el año 2001, exhibición de danzas urbanas a cargo del grupo Swingdigentes en 2015 y un concurso de baile organizado por la Orquesta Syberia en 2012.

\section{CONCLUSIONES}

A lo largo de las tres décadas en las que hemos realizado nuestro análisis documental, hemos podido constatar la presencia de más de 154 agrupaciones de

21. Durante los cinco días de las fiestas patronales, uno de ellos se traslada la patrona local en romería hacia el barrio de Los Cortijos, perteneciente a Fuente Librilla. Allí se suceden distintas actividades musicales y religiosas.

22. Jesús Moya, natural de Mula.

23. Acompañados por el grupo de batucada Timbaloe. En el 2017 se hace mención a otra batucada que recoge a los niños del colegio y realizan un desfile, pero no se menciona nombre. 
diversa índole relacionadas con la música. Principalmente de grupos u orquestas musicales para amenizar la verbena popular y propiciar el baile. Es notoria la cantidad de géneros y estilos de música que convergen durante cinco días en un mismo lugar, proporcionando a sus oyentes un amplio espectro de la cultura musical, al menos desde una perspectiva de la recepción auditiva.

Desde el punto de vista social, la actividad musical en Fuente Librilla durante sus fiestas patronales desempeña multitud de roles posibles. Desde fomentar el baile y la escucha como ocio; servir de acompañamiento para actividades religiosas y solemnes; amenizar concursos, juegos y actividades de diversa índole; despertar a la población o entretener a los habitantes de diferentes edades; hasta hacer de la fiesta un elemento esencial indispensable.

Las fuentes utilizadas que nos han servido para llevar a cabo nuestro estudio son el único documento escrito hasta la fecha que permite dejar constancia de la evolución de la actividad musical que vive, participa y forma parte de ella la sociedad de Fuente Librilla. Es por ello que con nuestro estudio también pretendemos poner en valor la necesidad de apreciar los documentos que autoproducimos y versan sobre nuestra forma de vida como sociedad, porque solo así seremos capaces de llevar a cabo investigaciones como esta que hacen posible el conocimiento a generaciones futuras de nuestra historia, en este caso de la actividad musical de un lugar.

\section{BIBLIOGRAFÍA}

\subsection{Programas de fiestas}

Comisión Organizadora. (1924). Fuente Librilla. Homenaje a su Excelsa Patrona en la subida solemne a su nuevo camarín. Murcia: Imprenta de Joaquín Abellán.

Comisión de Festejos. (1944). Fiestas Cívico-Religiosas en la Aldea de Fuente Librilla. Durante los dias 7, 8 y 9 de octubre de 1944 en honor de su Excelsa Patrona la Santísima Virgen del Rosario. Fuente Librilla: Comisión de Festejos.

Comisión de Festejos. (1946). Fiestas Cívico-Religiosas en la Aldea de Fuente Librilla. Durante los días 5, 6 y 7 de octubre de 1946 en honor de su Excelsa Patrona la Santísima Virgen del Rosario. Mula: Imprenta Victoria.

Comisión de Festejos. (1950). Fiestas Cívico-Religiosas en la Aldea de Fuente Librilla en honor a la Stma. Virgen del Rosario durante los días 7, 8 y 9 de octubre de 1950. Mula: Imprenta Victoria.

Comisión de Festejos. (1957). Fuente Librilla. Fiestas Cívico-Religiosas en honor a la Stma. Virgen del Rosario. Año 1957. Fuente Librilla: Comisión de Festejos.

Comisión de Fiestas. (1982). Fuente Librilla 82. Fiestas a celebrar durante los dias 9, 10, 11 y 12 de octubre en honor de nuestra Patrona Santísima Virgen del Rosario. Del 5 al 9 de octubre. Fuente Librilla: Imprenta Victoria. 
Comisión de Fiestas. (1984). Fuente Librilla 1984. Fiestas a celebrar durante los días 12, 13, 14 y 15 de octubre en honor de nuestra Patrona la Santísima Virgen del Rosario. Fuente Librilla: Imprenta Victoria.

Comisión de Fiestas. (1986). Fuente Librilla en honor de Ntra. Sra. Stma. Virgen del Rosario del 4 al 7 de octubre 1986. Fuente Librilla: Imprenta Alhameña.

Comisión de Fiestas. (1988). Fuente Librilla. Fiesta en honor de Ntra. Sra. Stma. Virgen del Rosario del 7 al 11 de octubre 1988. Fuente Librilla: Imprenta Alhameña-Pemán.

Comisión de Fiestas. (1989). Fuente Librilla. Fiestas en honor de Ntra. Sra. Stma. Virgen del Rosario del 6 al 10 de octubre de 1989. Fuente Librilla: Comisión de Fiestas.

Comisión de Fiestas. (1990). Ntra. Sra. del Rosario. Patrona de Fuente Librilla. Fiestas Patronales 1990. Murcia: Imprenta Gallego.

Comisión de Fiestas. (1991). Stma. Virgen del Rosario. Del 4 al 8 de octubre de 1991. Fuente Librilla: Comisión de Fiestas.

Comisión de Fiestas. (1992). Ntra. Sra. del Rosario. Patrona de Fuente Librilla. Fiestas Patronales 1992. Murcia: Imprenta Gallego.

Comisión de Fiestas. (1993). Fuente Librilla en Fiestas. Del 8 al 12 de octubre de 1993. Fuente Librilla: Comisión de Fiestas.

Comisión de Fiestas. (1994). Fiestas patronales en Fuente Librilla en honor a la Stma. Virgen del Rosario. Del 7 al 11 de octubre de 1994. Fuente Librilla: Comisión de Fiestas.

Comisión de Fiestas. (1995). Feria y Fiestas Fuente Librilla 95. Fuente Librilla: Comisión de Fiestas.

Comisión de Fiestas. (1996). Programa de Fiestas Fuente Librilla. Del 4 al 8 de octubre de 1996. Fuente Librilla: Comisión de Fiestas.

Comisión de Fiestas. (1997). Fuente Librilla 1997. Fiestas Patronales. Del 3 al 7 de octubre. Fuente Librilla: Comisión de Fiestas.

Comisión de Fiestas. (1998). Fuente Librilla. Fiestas Patronales. En honor a nuestra Patrona Ntra. Sra. Virgen del Rosario. Del 9 al 13 de octubre de 1998. Fuente Librilla: Comisión de Fiestas.

Comisión de Fiestas. (1999). Fiestas Patronales. En honor a nuestra Patrona Stma. Virgen del Rosario. Fuente Librilla. Del 9 al 13 de octubre de 1999. Murcia: Imprenta Gallego.

Comisión de Fiestas. (2000). Fiestas Patronales en honor a nuestra patrona Stma. Virgen del Rosario. Fuente Librilla del 6 al 10 de octubre 2000. Fuente Librilla: Comisión de Fiestas.

Comisión de Fiestas. (2001). Fiestas Patronales en honor a nuestra patrona Stma. Virgen del Rosario. Fuente Librilla del 5 al 9 de octubre 2001. Fuente Librilla: Comisión de Fiestas.

Comisión de Fiestas. (2002). Fiestas €uro-2002 Fuente Librilla. En honor a nuestra patrona Santísima Virgen del Rosario. Del 4 al 8 de octubre. Fuente Librilla: Comisión de Fiestas.

Comisión de Fiestas. (2003). Programa de Actos y Fiestas Patronales. Fuente Librilla 2003. En honor a la Santísima Virgen del Rosario. Del 26 de septiembre al 7 de octubre. La fiesta está en la calle. La comisión está contigo. Fuente Librilla: Comisión de Fiestas. 
Comisión de Fiestas. (2004). Programa de Actos y Fiestas Patronales en Honor a la Stma. Virgen del Rosario del 7 al 12 de octubre 2004. Fuente Librilla: Comisión de Fiestas.

Comisión de Fiestas. (2005). Fiestas Patronales Fuente Librilla 2005. En Honor a la Stma. Virgen del Rosario del 7 al 11 de octubre. Fuente Librilla: Comisión de Fiestas.

Comisión de Fiestas. (2006). Fiestas Patronales en Honor a la Virgen del Rosario. Fuente Librilla del 6 al 10 de octubre 2006. Fuente Librilla: Comisión de Fiestas.

Comisión de Fiestas. (2007). En honor a Ntra. Sra. del Rosario Fiestas Patronales Fuente Librilla 2007 del 5 al 9 de octubre. Fuente Librilla: Comisión de Fiestas.

Comisión de Fiestas. (2008). Fiestas Patronales Fuente Librilla 2008 en honor a la Stma. Virgen del Rosario del 3 al 7 de octubre. Fuente Librilla: Comisión de Fiestas.

Comisión de Fiestas. (2009). Programa de Fiestas Patronales 2009. En honor a Ntra. Sra. del Rosario. Los días 9, 10, 11, 12 y 13 de octubre. ;Una fuente de ilusión! Fuente Librilla: Comisión de Fiestas.

Comisión de Fiestas. (2010). En honor a Ntra. Sra. del Rosario Fiestas Patronales Fuente Librilla 2010 8-9-10-11 y 12 de octubre. Fuente Librilla: Comisión de Fiestas.

Comisión de Fiestas. (2011). Fiestas Patronales Fuente Librilla 2011. En honor a la Stma. Virgen del Rosario. Del 5 al 9 de octubre. Fuente Librilla: EdgSBM.

Comisión de Fiestas. (2012). Fiestas Patronales Fuente Librilla 2012. En honor a la Stma. Virgen del Rosario. Del 5 al 9 de octubre. Fuente Librilla: Comisión de Fiestas.

Comisión de Fiestas. (2014). Fiestas 2014 del 3 al 7 de octubre Fuente Librilla. Ntra. Sra. Virgen del Rosario. Fuente Librilla: Comisión de Fiestas.

Comisión de Fiestas. (2015). Fiestas Fuente Librilla 2015 del 7 al 13 de octubre. Ntra. Sra. Virgen del Rosario. Fuente Librilla: Comisión de Fiestas.

Comisión de Fiestas. (2016). Fiestas Patronales Fuente Librilla. En honor a la Stma. Virgen del Rosario. Fuente Librilla. 7, 8, 9, 10 y 11 octubre 2016. Fuente Librilla: Comisión de Fiestas.

Comisión de Fiestas. (2017). Fuente Librilla del 5 al 9 de octubre Fiestas 2017 en honor a Ntra. Sra. Virgen del Rosario. Fuente Librilla: Comisión de Fiestas.

Comisión de Fiestas. (2018). Fiestas Patronales Fuente Librilla en honor a Ntra. Sra. del Rosario. Octubre 6, 7, 8, 9 y 10 Fuente Librilla 2018. Fuente Librilla: Comisión de Fiestas.

Comisión de Fiestas. (2019). 2019 Fiestas Fuente Librilla del 4 al 8 de octubre en Honor a Ntra. Sra. Virgen del Rosario. Fuente Librilla: Comisión de Fiestas.

\subsection{Referencias}

Aramburu Urtasun, M. (1987). El baile en las fiestas patronales de los pueblos de Erroibar y Artzibar. Cuadernos de Etnología y Etnografía de Navarra, 19(49), 39-60. http:// www.culturanavarra.es/uploads/files/03_CEEN49_0039-0060.pdf

Barquero Caballero, F. J. (2017). El legado musical de la familia Espada en Librilla. En R. Montes Bernárdez (Coord.), Historia de las bandas de música de la Región de Murcia (pp. 383-394). Murcia: Asociación de Cronistas Oficiales de la Región de Murcia. 
Botella Nicolás, A. M. (2015). La música festera en la Revista de Fiestas de Moros y Cristianos de Alcoy (1940-1970): un análisis documental (I). Revista de Folklore, 400, 25-46. https://funjdiaz.net/folklore/07ficha.php?ID=4003\&NUM=400

García Domene, J. C. (2003). Anotaciones y curiosidades del templo parroquial. En Comisión de Fiestas (Ed.), Programa de Actos y Fiestas Patronales. Fuente Librilla 2003. En honor a la Santísima Virgen del Rosario. Del 26 al 7 de octubre. La fiesta está en la calle La comisión está contigo (pp. 24-27). Fuente Librilla: Comisión de Fiestas.

García Martínez, T. (2012a). Fuentes informativas para el estudio de las fiestas tradicionales de invierno en el sureste peninsular: (1897-1903) (tesis doctoral). Universidad de Murcia. Murcia. https://digitum.um.es/digitum/handle/10201/28787

García Martínez, T. (2012b). Noticias Históricas de Fuente Librilla a través de la prensa escrita. En Comisión de Fiestas (Ed.), Fiestas Patronales Fuente Librilla 2012. En honor a la Stma. Virgen del Rosario. Del 5 al 9 de octubre (p. 37). Fuente Librilla: Comisión de Fiestas.

López Espín, J. (2017). Aproximación histórica de la banda municipal de música de Mula. En R. Montes Bernárdez (Coord.), Historia de las bandas de música de la Región de Murcia (pp. 439-451). Murcia: Asociación de Cronistas Oficiales de la Región de Murcia.

López Núñez, N. (2012). Los auroros de Fuente Librilla. Murcia: Diego Marín.

López Núñez, N. (2015a). Cancionero popular de Fuente Librilla. Málaga: Editorial Monsálvez S. L.

López Núñez, N. (2015b). Fuente Librilla y su identidad musical. La Falcata, 3, 8.

López Núñez, N. (2016). De la música al crimen. Apuntes para una posible historia de Fuente Librilla. La Falcata, 5, 10.

P. (10 de octubre de 1900). En Fuente Librilla. Las Provincias de Levante, p. 1.

Sánchez Riquelme, F. (2017). Historia de las bandas de música en Alcantarilla. En R. Montes Bernárdez (Coord.), Historia de las bandas de música de la Región de Murcia (pp. 79-90). Murcia: Asociación de Cronistas Oficiales de la Región de Murcia.

Valverde García, M. (2017). Bandas de música en las fiestas de Campos del Río. En R. Montes Bernárdez (Coord.), Historia de las bandas de música de la Región de Murcia (pp. 195-206). Murcia: Asociación de Cronistas Oficiales de la Región de Murcia. 